\title{
Technological machines, construction resources, efficiency and safety
}

\author{
Nadegda Sevryugina* and Pavel Kapyrin
}

Moscow State University of Civil Engineering, 26, Yaroslavskoe Shosse, Moscow, Russia, 129337

\begin{abstract}
Understanding the direct relationship of the person, his activities Wednesday habitats requires not only new methodological approaches to research, but, first and foremost, clarify the existing scientific theories, their coherence with the development the environment Wednesday. A model of modernization of transport and technological machines as a factor in the realization of tasks of improving their effectiveness, safety and environmental performance. The main focus in addressing modifications of technological machines in use is considered lowering their material intensity, by adopting a more rugged and durable in endurance and wear materials. Modified methodology of estimation of residual resource of technical facilities by implementing technology upgrades for further reliable and safe operation. In the method a key factor is the effective and safe operation. Developed a calculation model of optimization of the resource in terms of assessing risk of system failure due to functional aging; optimization model of the resource, frequency of maintenance and wear limit of units in terms of assessing risk of occurrence of a system failure affecting safety. The solution of the question of resource upgrading to provide manufacturers, as an additional stage of the life cycle of construction machinery and means of complex mechanization.
\end{abstract}

\section{Theoretical and practical background}

Until now, natural resources are used for human needs, and sometimes just subjected to destruction when addressing the varying industrial tasks. Hastiness action led to a different kind of man-made disasters, after which world powers began to pay attention to the consequences that entails flying human thought in an effort to not only learn, but unknown and conquer yourself in serve the surrounding wednesday and its phenomena.

In its development humanity uses both external and internal reserves. Note that the external reserves are natural resources, usually not renewable, internal we can assume human intellectual capabilities are unlimited, and social society and the standard of living, to which is humanity, only confirm this fact.

As a result of years of research, based on an in-depth analysis of the development of scientific knowledge is that the fundamental laws of nature and technology are simple in

\footnotetext{
${ }^{*}$ Corresponding author: nssevr@yandex.ru
} 
form and variation in its basis. The more common is the Act, the easier it is mathematical expression and the easier it is to give it a variable interpretation $[1-4,5]$.

Before embarking on the research of technical systems, it is necessary to harmonize the terminology concept, rationalize their target function.

If we take into account the fact that in nature everything is connected, the person's ability to objectively perceive the different nature of the events has a significant impact on the final result of this relationship.

The term event refers to what was, is or will be. The content and structure of an event can be any. The only limitation is the completeness of events in at least some respects (indifferent any), so it should be treated as a whole.

Events do not recur, but may be identical to each other. Each event contains two sides: process (status change) and its substrate-what happened (is happening, will happen) this process $[1,6]$.

In the most general case, the process is characterized as state change its substrate. All mathematical dependences describing the quantitative aspect of process structure, the argument provides time (or single-valued functions of time).

Every mechanical movement includes three main components: the cause-effect $\boldsymbol{P}$, moving the $\boldsymbol{x}$, time $\boldsymbol{t}$. Traffic law sets the ratio between these values in the form of differential equations $[2,5]$.

Even in earlier writings I. Prigozhina and his school says that their [2] for irreversible processes now it is proved that all development of the universe is an irreversible process [1], reversible processes correspond to stable as substrates on which it is based processes.

Presentation of the fact that all processes occurring in the fields allows us to establish a deep connection of the above variables, generalized mechanics with geometry.

All of the above helped clarify the notions of reversibility and irreversibility [3]:

- reversible process called his state change of substrate, which can spontaneously go in both "live" and "reverse" directions on the same track;

- irreversible processes can go back on the same path, and "forward" only under the influence of external factors.

\section{Methods of resource regulation, security, technological machines within the structure of regional economic development}

The concept of "sustainability technical level of life support the region" includes objects of technical support, including in the separate category should be allocated to the class of building machines and means of complex mechanization - construction machines and tools integrated mechanization (CM@T).

The objective function of different types of construction machines, laying a manufacturer focused on performing the individual processes that are common to different regions with their livelihood, in particular: in the construction of buildings, roads, their maintenance and utilization [4-6].

The analysis revealed that in the coming years, the question remains maintain the health and safety of CM@T [7-9]. Given the scale and cost of solving the problem, proposes an alternative based on the methodology for the identification and implementation of residual life of technical means for human life support through the implementation of technology modernization, ensuring their continued reliable operation.

From a scientific point of view, the resource CM@T laid down by the manufacturer for the period of operation is reduced and is quantified by the integral value, including the amount of residual resources throughout the circuitry included in the system design. 
Accordingly, CM@T represent a technical system, the element base which is characterized by a random state changes in time, i.e., possessing the characteristics of uncertainty.

To minimize the effect of the time factor is provided by the system of technical maintenance and repair, which is currently quite worked out at normative and technological level allows to support the resource performance in accordance with the technical requirements laid down by the manufacturer.

Developed and introduced in practice of repair facilities recovery methods allow not only $100 \%$ to recover the properties of worn surfaces of the parts, but sometimes, and increase values of properties compared to the original through the use of new technologies and materials.

Because the purpose of repair effects is the maintenance or restoration of the resource (increase in the operational stage of the life cycle of the machine), then such actions can be characterized as resource modernization, aims to minimize the influence of the time factor and maintain the pledged level of quality of the machine.

The analytical results and using the basic application of probability theory generated a number of distributions showing the structure studied, CM@T [7, 10], what can be used structurally and effect or functional block diagram.

Methods of resource modernization is presented in the following stages:

- inspection of facilities-finding of the residual resource and the preparation of structural schemes of reliability;

- assessment of the risk of failure of hardware components for the hierarchical levels of security;

- assessment of the level of moral and physical ageing;

- development of a list of modernization measures with rationale in terms of cost and safety;

- information-methodical and technological support of the project to ensure it is operational prior to the date of disposal.

To assess the safety of the restored functionality, $\mathrm{CM} @ \mathrm{~T}$ after resource of modernization in the system of human life support in the event of a dangerous situation caused by the failure of the system and performed the service impact the elimination of their consequences, you can try typing the term " resource after minor repairs " $\left(T_{R m r}^{\prime}\right)$ and the indicator of cost elimination of failures and malfunctions for a separate operational period:

$$
C_{m r}=\frac{e}{\gamma+1} T^{\gamma+1}
$$

Where $e, \gamma$ - empirical coefficients.

Seems to be a few variants of this model:

- model of resource optimization in terms of assessing risk of system failure due to functional aging of the circuitry.

The target function this model is represented in value terms and takes the form:

$$
C(T)=\frac{\vartheta_{\text {tar.f }}(T)}{M}\left[\frac{C_{d e p}}{T_{m r}^{\prime}}+\frac{e}{\gamma+1}\left(T_{m r}^{\prime}\right)^{\gamma}\right]+\sum_{i} \frac{C_{w c i}}{T_{w c i}} k_{\kappa . c .} \rightarrow \min .
$$

Calculation of optimal resource for repair of impacts includes the base cost of the machine, the values of empirical indicators to the largest maintenance costs: 


$$
T_{\text {or. }}=\left(\frac{C_{B}}{\vartheta_{\text {tar.f }}(T)} \frac{(\gamma+1)}{e \gamma}\right)^{\frac{1}{\gamma+1}}
$$

-model of resource optimization, maintenance intervals and wear limit assembly units in terms of assessing risk of occurrence of a system failure affecting safety.

The optimization model of the resource, frequency of maintenance and wear limit, the objective function of this model takes the form:

$$
C(T)=\frac{\vartheta_{t a r . f}(T)}{M}\left[\frac{C_{d e p}}{T_{m r}^{\prime}}+\frac{e}{\gamma+1}\left(T_{m r}^{\prime}\right)^{\gamma}+k_{S W} k_{m} b^{\psi} T_{d e p}^{(\alpha-1) \psi} T_{m r}^{\psi-1}\right]+\sum_{i} \frac{C_{w c i}}{T_{w c i}} k_{q s} \rightarrow \min
$$

Estimated resource machine residual formula becomes:

- operation residual resource

$$
C_{\text {res.res }}=\frac{C_{B}\left(T_{B}-T_{o p}\right)}{T_{B}} e^{-K_{\Sigma} \cdot \psi}
$$

Where $e$ is the base of natural logarithms, $e \approx 2,72$;

$K_{\Sigma}$ - coefficient taking into account the various factors affecting the value of physical deterioration;

$\psi$ is a function depending on age and experience, $\mathrm{CM} @ \mathrm{~T}$ since the beginning of operation.

\section{Example of calculation of statistics}

In the example substantiates the performance of resource upgrading and development for these purposes the set of activities performed for the road grader.

In the calculation of the accepted value established by the manufacturer of the amortization term of the service (recommended $8 . . .10$ years, depending on engine power, the calculations made 9 years) and annual achievements, with the introduction of a lower limit probability the functionality of the machine, equal to $0.80(\gamma=80 \%)$.

Used to calculate the indicators: full average service life, thousand hour; the rounded value of secondary resource, multiple maintenance periodicity, thou hours; average resource to first overhaul.

At the request of the customer the value of the probability functionality can be increased, Figure 1.

The value of the secondary resource are rounded to the nearest multiple of the periodicity of maintenance of the machine. The obtained curves show the minimum value of normalized resource of the machine to the adopted design values. The curve of the resource step. Intermittent double line indicates changes of a resource, characterizing the dependence between the annual operating time of the machine and the underlying resource.

It is necessary to note that in the calculations are obtained the average values of the resource that should guide the operator as it becomes the main factor of uncertainty because it is not economically profitable to work in terms of the averaged main indicators of reliability of the machine.

The average value of the resource machine does not allow to evaluate the resource of basic knots and units. 


\section{Discussion}

Production conditions, which are economical to use machines represent the area of their rational application. In turn, the field of rational application of the machine represents a zone of change of the parameters characterizing the conditions of operation of the machine in which this machine compared to others provides the greatest economic efficiency.

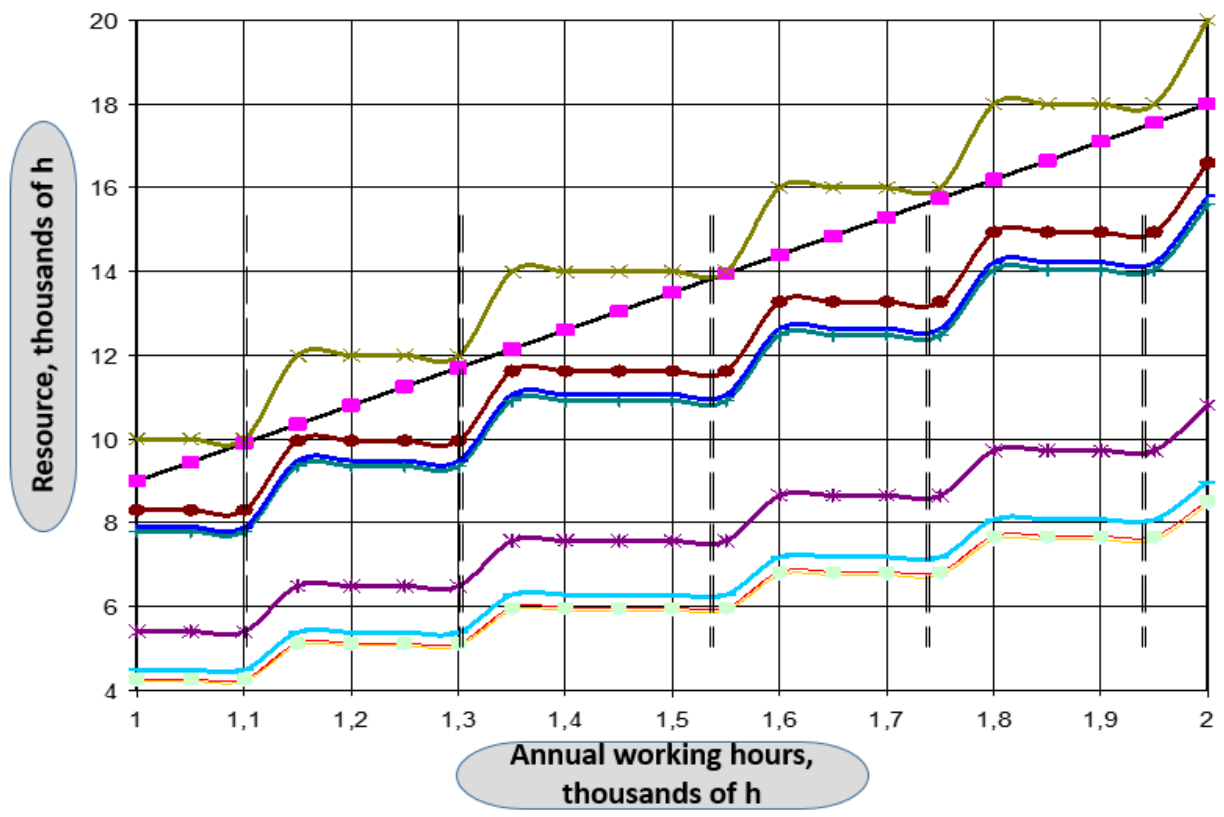

Fig. 1 Dynamics of change of a given machine resource technologies maintain, on an example of motor grader.

Construction and road machines are at increased risk and their safety has become increasingly costly over the years.

The high cost of production of new machines poses the problem of modernization of already operated machines. The proposed system-resource approach allows to improve the efficiency of machines taking into account the development of technology.

With a sufficient degree of certainty it can be argued that the target modernization provides for expansion of the field of rational use of machinery during their operation and improves their effective use.

\section{Conclusions}

It has been established that the time factor, as characteristic of the machine life cycle changes its status to be monitored by minimizing its impact, which can be provided by the organizational-technological actions of various kinds modernization: the resource, functional and moral.

The proposed method of modernization of the resource CM@T ensure their reliable and safe operation.

Developed a calculation model of optimization of the resource in terms of assessing risk of failure due to functional aging of the system; the optimization model of the resource, frequency of maintenance and wear limit Assembly units in terms of assessing risk of occurrence of a failure affecting the safety. 
It is established that estimates the resource of modernization require a value, determined on the basis of the developed software systems, ensuring their monitoring at the operational stage of the life cycle of the machine.

Offered to machine, the question of resource upgrades, as an additional stage of the life cycle of construction machinery and means of complex mechanization.

\section{References}

1. M. V. Bunin, Kiev: NMCO, (1992)

2. I. Prigozhin, Sciences, M.: Nauka, (1985)

3. V. A. Zorin, N. S. Sevryugina and A. N. Daugello, (Belgorod: Publ. house BGTU im. V. G. Shukhov, 2009)

4. N. S. Sevryugina Const. and road mach. 7. 28-29 (2007)

5. A. V. Goropashnaya (Herald of St. Petersburg University, (2009)

6. P. D. Kapyrin and C. E. Romanova. Vestnik MGSU, 4-1,165-170 (2010)

7. V. G. Popov,, Cherepovets: State educational institution of CSU, (2005)

8. V. I. Balovnev and others Road-building machines and systems, Moscow-Omsk: Publishing house SibADI, (2001)

9. V. A. Zorin Bases of operability of technical systems, LLC Magistr-Press, (2005)

10. R. R. Sharapov, P. D. Kapyrin, S. Yu. Lozovaya, V. V. Yadykina and A. M. Agarkov. MATEC Web Conf., EDP Sciences. 86, 03007 (2016) 